\title{
A Direct Model Predictive Control Strategy for High-Performance Synchronous Reluctance Motor Drives
}

\author{
Jacopo Riccio, Student Member, IEEE, Petros Karamanakos, Senior Member, IEEE, Shafiq Odhano, Member, IEEE, \\ Mi Tang, Member, IEEE, Mauro Di Nardo, Member, IEEE, and Pericle Zanchetta, Fellow, IEEE
}

\begin{abstract}
This paper presents a finite control set model predictive control (FCS-MPC) method that improves the performance of a synchronous reluctance machine drive. As shown, when a high sampling-to-switching frequency ratio is used with FCSMPC, the stator current distortions can be significantly reduced, allowing for less losses in the machine. Moreover, the FCSMPC steady-state performance is enhanced by introducing an integrating element into the cost function to ensure accurate output reference tracking. Finally, the adopted drive model that relies on an identified accurate magnetic model of the machine further improves the robustness of the discussed control scheme. The presented simulation and preliminary experimental results verify the effectiveness of the discussed method.
\end{abstract}

\section{INTRODUCTION}

Synchronous reluctance (SyRel) machine drives are already employed in many industrial applications as valid alternatives to conventional drives based on induction and permanent magnet synchronous motors (PMSMs). The utilization of SyRel in many fields is justified by economical and performance reasons. For example, the simple rotor structure made by an iron core, shaped such that its working principle is based on the reluctance phenomenon, is cheap and easy to manufacture, and guarantees negligible rotor losses [1]. Moreover, the absence of winding and rare-earth permanent magnets mounted on the rotor makes the cost of the SyRel machines less prone to the price variability of the raw materials, and high rotational speeds are reachable. The downsides, however, of SyRel motors relate to the associated low power factor, the highly non-linear magnetic behavior [2], and the inherent torque ripple.

To tackle the last two issues, field oriented control (FOC) based on proportional-integral (PI) controllers is most commonly used [3]. Nevertheless, such a control method is not the most suitable solution for a non-linear system as a SyRel

Jacopo Riccio, Mi Tang, Mauro Di Nardo, and Pericle Zanchetta are with the Department of Electrical and Electronic Engineering, University of Nottingham, Nottingham, UK; e-mail:Jacopo.Riccio1@nottingham.ac.uk, mi.tang@nottingham.ac.uk, mauro.dinardo4@nottingham.ac.uk pericle.zanchetta@nottingham.ac.uk

Petros Karamanakos is with the Faculty of Information Technology and Communication Sciences, Tampere University, 33101 Tampere, Finland; email: p.karamanakos@ieee.org

Shafiq Odhano is with the School of Engineering, Newcastle University, Newcastle Upon Tyne, UK; e-mail: shafiq.odhano@newcastle.ac.uk drive, since its performance significantly varies according to the operating point and might lead to stability issues if a proper offline tuning procedure is not considered. Direct torque control (DTC) is another possibility to control SyRel drives [4], even though it is more prone to high flux and torque ripples.

An interesting alternative to the aforementioned control techniques is model predictive control (MPC), especially in its form as direct controller, known as finite control set MPC (FCS-MPC) [5]. Such a control strategy can effectively deal with complex, constrained, non-linear systems such as SyRel motor drives. FCS-MPC is nowadays particularly relevant thanks to the readily available hardware and software solutions that can deal with the associated underlying computational complexity of MPC algorithms, which used to be the main barrier to its adoption in the past. FCS-MPC is conceptually simple since the quality of the system behavior is quantified by a cost function that captures the control objectives. This function is evaluated for each one of the possible switch positions of the converter, and the one resulting in the minimum cost is selected and applied to the converter.

As can be seen in literature [6], most commonly the cost function of FCS-MPC takes into account the weighted reference tracking error terms [7]. However, as explained in [6], lack of penalization of the control effort can lead to poor performance due to the low switching granularity, i.e., low sampling-to-switching frequency ratio. The control effort factor was considered in several works aimed to minimize the switching losses as in [8], where the change of the switching state was penalized to reduce the commutation losses of a matrix converter. The same approach was adopted in [9] and [10] to control a PMSM and a SyRel, respectively. In [10], particular focus was given to the prediction horizon to enhance the MPC performance at steady state, even though just simulation results were provided. For this reason, in this work, conventional FCS-MPC is revisited, and special attention is paid to the impact of the control weighting factor on the cost function to improve the steady-state performance of the drive, while not deteriorating its dynamic behavior.

Another typical issue inherent to model-based control strategies, such as FCS-MPC, is the usually inaccurate and/or incomplete knowledge of the model of the system, leading to 
steady-state errors that can adversely affect the reference tracking performance of the controller [11]. As far as SyRel drives are concerned, an accurate identification of the magnetic model and inverter non-linearities is needed. Several approaches have been employed to tackle the model inaccuracies such as in [12], where the adoption of an online least-squares system identification can significantly improve the reference tracking behavior of MPC. In [13], an integral term was included in the cost function to deal with potential steady-state errors. The latter approach is the one followed in this work due to its simplicity and straightforward adaptability.

With an aim to achieve the desired performance through the adoption of FCS-MPC, two severe hardware requirements have to be met. First, the computational power required by the controller has to enable the real-time execution of the control algorithm. This is addressed thanks to the readily available cheap digital control platforms based on field programmable gate arrays (FPGAs), and digital signal processing (DSPs) that can easily deal with demanding algorithms such as FCSMPC. In addition, the power modules of the driving converter have to guarantee operations at high instantaneous switching frequencies, preserving the integrity of the devices, e.g., by employing silicone-carbide ( $\mathrm{SiC}$ ) power switches. To this end, sophisticated configurations to keep the operating temperature within the devices safe operating area while operating at very high switching frequencies were presented in [14] and [15].

The paper is structured as follows. The SyRel and the twolevel voltage-source inverter (2L-VSI) models used for the controller implementations are presented in Section II. The derivation of the FCS-MPC algorithm is provided in Section III. Moreover, in Section IV, simulation and experimental results are included that verify the superior performance of the proposed FCS-MPC algorithm when compared with the conventional one. Finally, conclusions and future works on this topic are provided in the last section of this paper.

\section{Synchronous Reluctance Machine Drive PREDICTION MODEL}

In this section the 2L-VSI and the SyRel mathematical models adopted for the implementation of the proposed control strategy are described.

\section{A. 2L-VSI Modelling}

The SynRel motor is driven by a SiC converter supplied by a voltage-controlled power source. The eight possible switch positions $(n)$ of the $2 \mathrm{~L}-\mathrm{VSI}$ form an hexagon in a stationary, orthogonal reference frame $(\alpha \beta)$, see Fig. 1 . The phase-voltage components are:

$$
\left\{\begin{array}{l}
v_{a}(n)=\frac{V_{d c}}{3}\left[2 S_{a}(n)-S_{b}(n)-S_{c}(n)\right] \\
v_{b}(n)=\frac{V_{d c}}{3}\left[2 S_{b}(n)-S_{a}(n)-S_{c}(n)\right] \\
v_{c}(n)=\frac{V_{d c}}{3}\left[2 S_{c}(n)-S_{a}(n)-S_{b}(n)\right]
\end{array}\right.
$$

where $V_{d c}$ is dc-link voltage set by the power supply, $S_{a}(n)$, $S_{b}(n)$, and $S_{c}(n)$ are the on-state (one), or off-state of the switch (zero), resulting in eight configurations whose values are listed in Table I. These configurations correspond to six
TABLE I: Switching states of the 2L-VSI.

\begin{tabular}{|c|c|c|c|c|c|c|c|c|}
\hline$n$ & 0 & 1 & 2 & 3 & 4 & 5 & 6 & 7 \\
\hline$S_{a}(n)$ & 0 & 1 & 1 & 0 & 0 & 0 & 1 & 1 \\
\hline$S_{b}(n)$ & 0 & 0 & 1 & 1 & 1 & 0 & 0 & 1 \\
\hline$S_{c}(n)$ & 0 & 0 & 0 & 0 & 1 & 1 & 1 & 1 \\
\hline
\end{tabular}

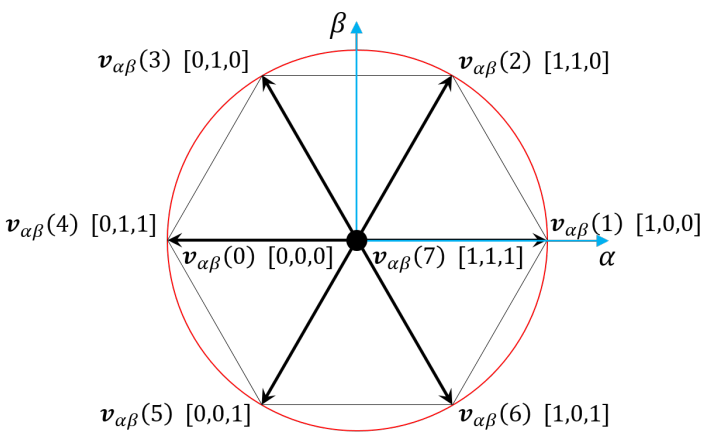

Fig. 1: 2L-VSI switching states.

active voltage vectors $\boldsymbol{v}_{\alpha \beta}(1,2 . .6)$, and two zero voltage vectors $\boldsymbol{v}_{\alpha \beta}(0), \boldsymbol{v}_{\alpha \beta}(7)$, as illustrated in Fig. 1 .

By using the following transformation, the $d q$-voltage vectors can be calculated as

$$
\boldsymbol{v}_{d q}(n)=\boldsymbol{P} \boldsymbol{v}_{a b c}(n)
$$

where $\boldsymbol{v}_{a b c}(n)$ is the phase-voltage vector, $\boldsymbol{P}$ is the rotation matrix combining the well-known Clarke and Park transformations as function of the electrical angle $\theta_{r}$, i.e.,

$$
\boldsymbol{P}=\frac{2}{3}\left[\begin{array}{lll}
+\cos \theta_{r} & +\cos \left(\theta_{r}-\frac{2 \pi}{3}\right) & +\cos \left(\theta_{r}+\frac{2 \pi}{3}\right) \\
-\sin \theta_{r} & -\sin \left(\theta_{r}-\frac{2 \pi}{3}\right) & -\sin \left(\theta_{r}+\frac{2 \pi}{3}\right)
\end{array}\right]
$$

In (2), the term $\boldsymbol{v}_{d q}(n)$ can be obtained from the 2L-VSI feasible configurations given in (1).

\section{B. SyRel Modelling}

FCS-MPC is based on the prediction of the state of the system, and it is crucial to have an accurate representation of the plant. The SyRel drive model used for the prediction of the currents is represented into the synchronous reference frame ( $d q$ coordinates) where the quantities can be assumed as constant vectors in a reference frame rotating with the electrical speed $\omega_{r}$. As the control algorithm is designed in the discrete-time domain, the discrete-time model of the drive is derived by employing forward Euler discretization as follows:

$$
\boldsymbol{i}_{d q}^{k+1}(n)=\boldsymbol{i}_{d q}^{k}+T_{s} \boldsymbol{L}^{-1}\left[\boldsymbol{v}_{d q}(n)-R_{s} \boldsymbol{i}_{d q}^{k}+\boldsymbol{Q} \omega_{r} \boldsymbol{\psi}_{d q}\right]
$$

On the left-hand side of (4), the superscript $k$ stands for the actual time step, while $k+1$ indicates the next time step, and $T_{s}$ is the sampling interval between the two samples. The term $\boldsymbol{i}_{d q}^{k+1}(n)$ represents the current prediction vector in $d q$ coordinates, calculated for each one of the possible switch positions $(n=0,1,2, \ldots, 7)$ of the $2 \mathrm{~L}$-VSI. The voltage vectors $\boldsymbol{v}_{d q}(n)$ are defined in (2); the term $\boldsymbol{i}_{d q}^{k}$ is the measured current vector; $\boldsymbol{\psi}_{d q}$ and $\boldsymbol{L}^{-1}$ are the magnetic flux vector and 


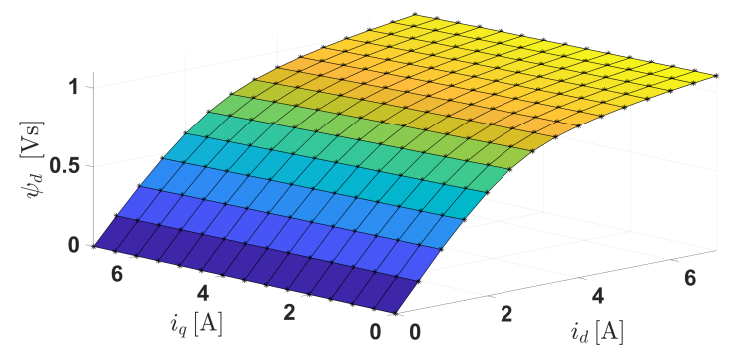

Fig. 2: d-axis magnetic model.

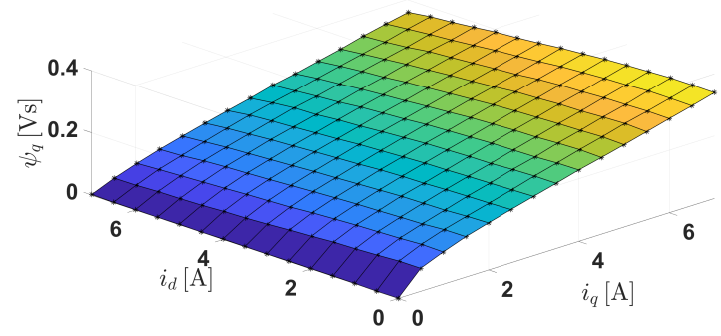

Fig. 3: q-axis magnetic model.

the inverse of the differential inductance matrix respectively, both assumed as functions of the current vector.

The peculiarity of the SyRel resides in the difference between the direct and the quadrature inductances terms $L_{d d}, L_{q q}$, respectively, to produce the electromagnetic torque. Moreover, the coupling inductance terms $L_{d q}, L_{q d}$ are nonzero, and are equal when the iron losses can be neglected at low speed operations. $Q$ is the orthogonal rotation matrix $\boldsymbol{Q}=\left[\begin{array}{cc}0 & -1 \\ 1 & 0\end{array}\right]$, and $R_{s}$ is the stator resistance.

To improve the performance of the controller developed in Section III, an accurate prediction model is required; due to the model-based nature of FCS-MPC, any model inaccuracies can adversely affect the predictions, and subsequently, the system behavior. Hence, non-linearities due to self-axis and cross-saturation phenomena need to be taken into account and effectively addressed. To this end, the magnetic model is identified by performing constant speed identification tests [16]. In doing so, the flux can be estimated and the maps that indicate the relationship between the current and the developed flux (see Figs. 2 and 3) can be stored as lookup tables (LUTs). From the given magnetic characteristics the maximum torque per Ampere (MTPA) trajectory is also estimated, but it is omitted in this work. The incremental inductance matrix $\boldsymbol{L}=\left[\begin{array}{cc}L_{d d} & L_{d q} \\ L_{q d} & L_{q q}\end{array}\right]$ is calculated as follows

$$
\begin{aligned}
& L_{d d}=\frac{\psi_{d+1}^{q}-\psi_{d}^{q}}{\Delta i} \\
& L_{q q}=\frac{\psi_{q+1}^{d+1}-\psi_{q}^{d}}{\Delta i} \\
& L_{d q}=\frac{\psi_{d+1}^{q+1}-\psi_{d}^{q}}{\Delta i} \\
& L_{q d}=\frac{\psi_{q+1}^{d}-\psi_{q}^{d}}{\Delta i}
\end{aligned}
$$

In (5), the term " $\Delta i$ " is the fixed current step used in the magnetic model identification, while subscripts identify the flux map, and superscripts used in the detected points lie on.

\section{Direct Model Predictive Control With REFERENCE TRACKING}

The current control of a SyRel machine drive is implemented by means of direct MPC with reference tracking, commonly referred to FCS-MPC. The main control objective of the controller is the accurate tracking of the current reference. This has to be achieved while reducing the switching frequency to keep the power switching losses low. However, the effectiveness of the controller greatly depends on the accuracy of the prediction model (4). This is due to the fact that FCS-MPC is, in essence, a proportional controller. Hence, any existing mismatches and system non-idealilities can amplify potential tracking errors. Considering that computationally demanding self-commissioning identification techniques and/or elaborate observers can further increase the computational load of the FCS-MPC method, in this work we introduce an integrating element into the optimization problem to eliminate potential steady-state errors.

Given the above, the cost function that can tackle all the aforementioned challenges needs to be formulated. To this end, the error between the references $i_{d}^{*}, i_{q}^{*}$ and the predictions components $i_{d}^{k+1}(n), i_{q}^{k+1}(n)$ at the next time step $k+1$ defined in (4), is defined as

$$
\boldsymbol{i}_{d q, \text { err }}^{k+1}(n)=\boldsymbol{i}_{d q}^{*}-\boldsymbol{i}_{d q}^{k+1}(n)
$$

while the current error at step $k$, i.e., $i_{d, e r r}^{k}, i_{q, e r r}^{k}$ is

$$
\boldsymbol{i}_{d q, e r r}^{k}=i_{d q}^{*}-i_{d q}^{k}
$$

The cost function $J(n)$ can be introduced as the sum of the (squared) 2-norm of the current components errors with the integrating term added to it, and an additional term representing the control effort, i.e.,

$$
\begin{aligned}
J(n) & =\left\|\boldsymbol{i}_{d q, e r r}^{k+1}(n)+\boldsymbol{W}_{i_{d q}} T_{s}\left(\sum_{j=1}^{m} \boldsymbol{i}_{d q, e r r}^{k-j}+\boldsymbol{i}_{d q, e r r}^{k}\right)\right\|_{2}^{2} \\
& +\lambda_{u}\|\Delta \boldsymbol{S}(n)\|_{2}^{2}
\end{aligned}
$$

The integrals of the actual current components errors are evaluated at every sample $(m)$, and the terms $i_{d q, e r r}^{k-j}$ are the previously calculated integral errors, and $\boldsymbol{W}_{i_{d q}}$ is the diagonal 
matrix whose nonzero entries are the two weighting factors introduced for the integral, tuned in a trial-and-error manner.

The control effort factor is defined as

$$
\Delta \boldsymbol{S}(n)=\boldsymbol{S}_{a b c}(n)-\boldsymbol{S}_{a b c}^{k-1}
$$

The term $\boldsymbol{S}_{a b c}(n)=\left[S_{a}(n) S_{b}(n) S_{c}(n)\right]^{T}$ is evaluated for each configuration of the 2L-VSI ([0,0,0], [1,0,0], ... [1,1,1]), while $\boldsymbol{S}_{a b c}^{k-1}$ is the three-phase switch position applied at the previous time step $k-1$. The control effort penalizes the change in the switch position, allowing to lower the average switching frequency, getting a more favorable granularity of switching, defined as the ratio between the sampling frequency $\left(f_{s}=1 / T_{s}\right)$ and the average switching frequency $\bar{f}_{s w}$, thus permitting operation in a quasi-continuous time domain, and achieving lower current distortions [6]. To achieve a high ratio, and thus fine switching granularity, the weighting factor $\lambda_{u}>0$ is added. Conventional FCS-MPC does not include the control effort penalization, or equivalently $\lambda_{u}=0$. This implies that the switching frequency is not directly controlled, but merely limited by the sampling frequency which defines a theoretical upper limit $\bar{f}_{s w}=f_{s} / 2$. Because of this, conventional FCS-MPC achieves a poor granularity of switching, which has a negative impact on the drive performance, as indicated in the next section. Hence, the aim is to have as high a sampling frequency as possible, and tune $\lambda_{u}$ such that the average switching frequency is reduced, and, consequently, a fine granularity of switching is achieved. Thanks to contemporary microprocessors that come with high computational power and low costs, a cost-effective real-time implementation of the proposed FCS-MPC approach is feasible, as also verified in Section IV.

The cost function defined in (8), selects the optimum vector in order to achieve the desired behavior, and sends the corresponding gate signals $S_{g}$ to the three legs of the 2L-VSI. In the feedback, two of the phase current components $\left(i_{b}, i_{c}\right)$ and the electrical angle are used to predict the current components according to (4) at the next sample time instant. The complete block diagram of the FCS-MPC method is presented in Fig. 4.

\section{Simulation and Experimental Results}

In this section the simulation and the experimental results of the FCS-MPC scheme for a SyRel machine driven by a 2L-VSI are performed to evaluate its performance. The steady-state performance metrics adopted here to evaluate the proposed FCS-MPC algorithm, are the average switching frequency $\bar{f}_{s w}$, and the current total demand distortion (TDD) shown in the equations below:

$$
\begin{aligned}
\bar{f}_{s w} & =\lim _{\ell \rightarrow \infty} \frac{1}{\nu \ell T_{s}} \sum_{z=0}^{\ell-1}\left\|\Delta \boldsymbol{S}_{a b c}(z)\right\|_{1} \\
\operatorname{TDD}_{i} & =\frac{\sqrt{\sum_{n \neq 1} i_{n}^{2}}}{I_{n}}
\end{aligned}
$$

Above, in the definition of $f_{s w}, \ell$ is the time step, and $\nu$ is the number of power switches of the used converter, which

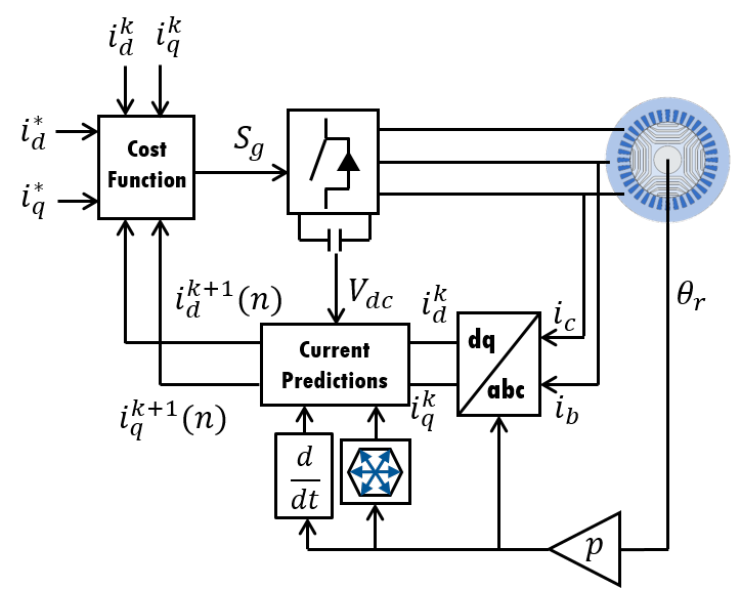

Fig. 4: Block diagram of FCS-MPC for a SyRel Motor drive.

\begin{tabular}{|c|c|c|c|}
\hline Variable & Symbol & Value & Unit \\
\hline Stator Resistance & $R_{s}$ & 3.15 & $\Omega$ \\
\hline Rated Torque & $T_{e m}$ & 14 & $\mathrm{Nm}$ \\
\hline Rated Speed & $\omega_{m}$ & 1500 & $\mathrm{rpm}$ \\
\hline Rated Power & $P_{n}$ & 2.2 & $\mathrm{~kW}$ \\
\hline Rated Current & $I_{n}$ & 5.5 & $\mathrm{~A}$ \\
\hline Rated Voltage & $V_{n}$ & 400 & $\mathrm{~V}$ \\
\hline Pole Pairs & $p$ & 2 & $\#$ \\
\hline
\end{tabular}

TABLE II: Table of values.

are six for a $2 \mathrm{~L}-\mathrm{VSI}$. The estimation of the average switching frequency in real time presents some difficulty. In this work, it is calculated from the instantaneous switching frequency as in (10a), by using a low-pass filter (LPF) considering the mean value over an integer number of electrical periods. The reason behind the use of a LPF is justified by the fact that communication issues might occur between the control platform and the host computer, especially with high sampling frequencies, and a down-sample is used during the data acquisition to limit the number of samples and facilitate the acquisitions.

Regarding the TDD definition, it is calculated by taking into account the energy spread over all harmonics except for the fundamental, and the denominator is based on the rated current $I_{n}$. It is also calculated in a post-processing stage over an integer number of electrical periods, and averaged over the three phases.

Finally, it should be noted that the controlled machine is in current-control mode, while the load machine is in speedcontrol mode to ensure that the rotational mechanical speed is fixed.

\section{A. Simulation Results}

A set of simulations are performed by varying $\lambda_{u}$, at various sampling frequencies, while keeping fixed the current references, the rotational speed, and the dc-link voltage. The current control trigger is performed within a time windows of $0.76 \mathrm{~s}$ lasting long enough to have a periodicity over one mechanical turn. The model of the SyRel considered included 


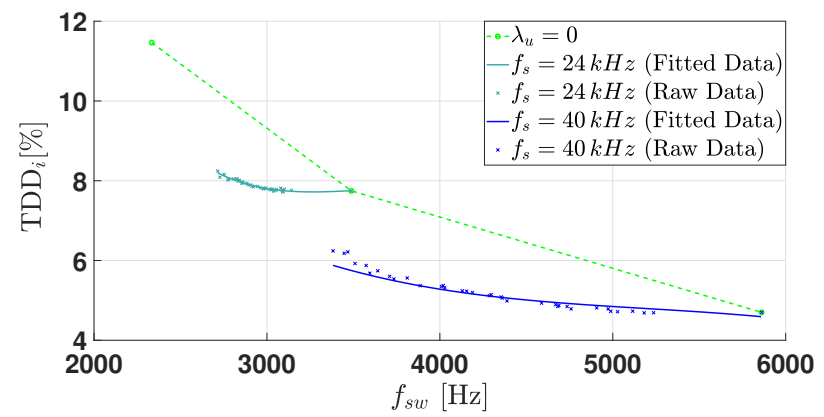

Fig. 5: Trade-off curve between current TDD and switching frequency at various $\lambda_{u}$ and full-load $\left(i_{d}=i_{q}=5.5 \mathrm{~A} ; \omega_{m}=1500 \mathrm{rpm}, 0<\lambda_{u}<\right.$ $0.056)$.
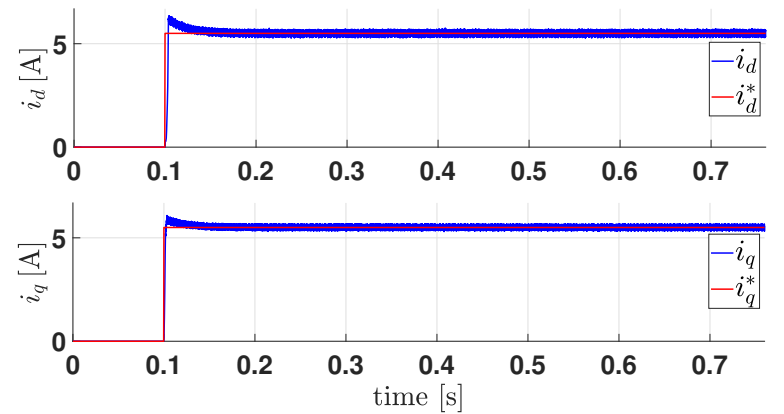

Fig. 6: $d q$-current reference tracking $\left(f_{s}=40 \mathrm{kHz}, \bar{f}_{s w} \approx 4020 \mathrm{~Hz}\right.$, and $\left.\lambda_{u}=0.0384\right)$.

the real magnetic model implemented as a LUT based on the curves given in Figs. 2 and 3. The drive model is based on (1), neglecting the effect of the dead-time.

The simulation results are shown at full load, where the mechanical speed is set to $1500 \mathrm{rpm}$ by the prime mover, while the current references are set to $5.5 \mathrm{~A}$ on both $d$ - and $q$-axis. The dc-link selected was set to $800 \mathrm{~V}$, in a way to guarantee the functioning within the saturation boundaries. The overall results are shown in Fig. 5 where it is noticeable a reduction of the current TDD for a given average switching frequency $\bar{f}_{s w}$ as the granularity of switching is improved. In the same figure the current TDD achieved with conventional FCS-MPC (i.e., $\lambda_{u}=0$ ) is shown. Note that to achieve a wide range of switching frequencies, the sampling frequency $f_{s}$ is adjusted accordingly. As can be seen, the proposed FCS-MPC clearly outperfoms the conventional one, thanks to the fine granularity of switching. The $d q$-current reference tracking is shown in Fig. 6, whereas the three-phase stator currents are shown in Fig. 7 for operation at $f_{s}=24 \mathrm{kHz}$ and $\bar{f}_{s w} \approx 4 \mathrm{kHz}\left(\lambda_{u}=\right.$ 0.0384 ). About $25 \%$ reduction in the TDD can be noticed at the same switching frequency.

\section{B. Experimental Results}

Experimental tests were also conducted to validate the proposed approach. The experimental rig (Fig. 8) consists of a prime mover coupled to the SyRel driven by an industrial

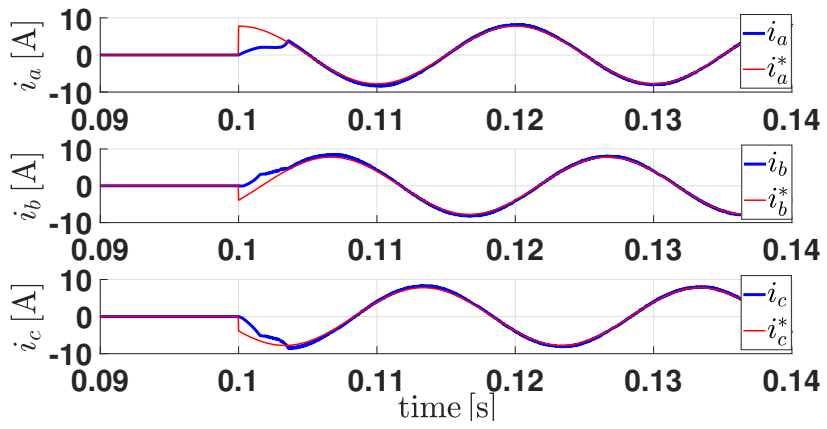

Fig. 7: $a b c$-current reference tracking $\left(f_{s}=40 \mathrm{kHz}, \bar{f}_{s w} \approx 4020 \mathrm{~Hz}\right.$, and $\lambda_{u}=0.0384, \mathrm{TDD}_{i} \approx 5.4 \%$ ).

drive (ID) sourced by a dc-power supply; the SyRel motor is driven by a $\mathrm{SiC} 2 \mathrm{~L}-\mathrm{VSI}$. The control platform employed to control both PM and SyRel is the one introduced in [17] where the gate signals are sent to the converters by optic signals. The two converters share the same dc-link. Two sets of experiments were conducted to show the effectiveness of the proposed control algorithm to guarantee zero steadystate reference tracking and a reduced TDD compared with conventional FCS-MPC. Due to the limitation in the dc-link voltage settings of the ID driving the prime mover, the full

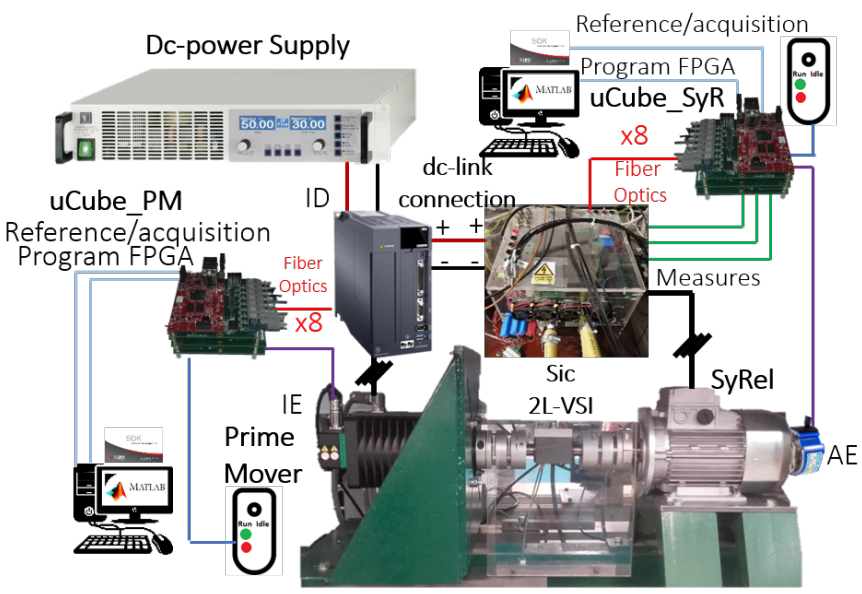

Fig. 8: Test bench configuration.

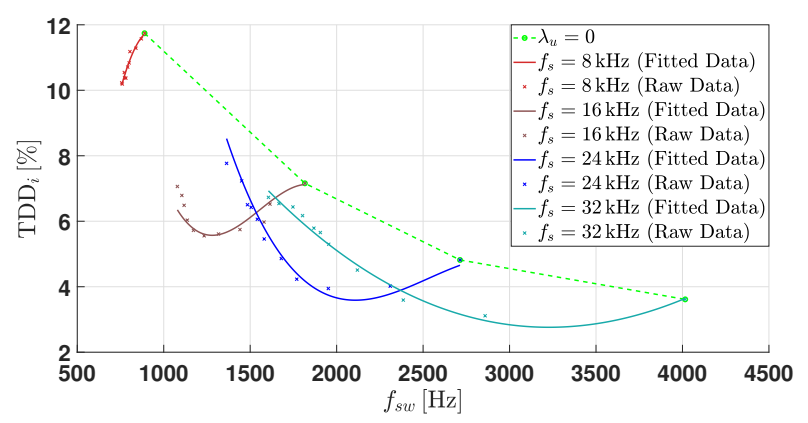

Fig. 9: Trade-off curve between current TDD and switching frequency at various $\lambda_{u}\left(i_{d}=i_{q}=1.1 \mathrm{~A} ; \omega_{m}=400 \mathrm{rpm}, 0<\lambda_{u}<0.08\right)$. 

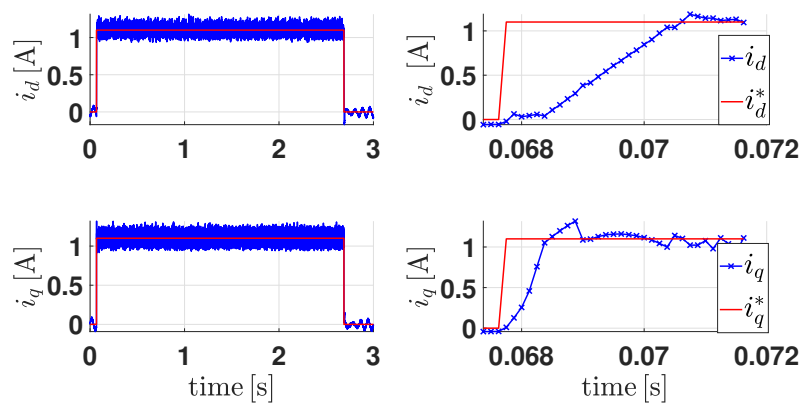

Fig. 10: $d q$-current reference tracking tracking $\left(f_{s}=24 \mathrm{kHz}, \bar{f}_{s w} \approx 2 \mathrm{kHz}\right.$, and $\left.\lambda_{u}=0.0160\right)$.

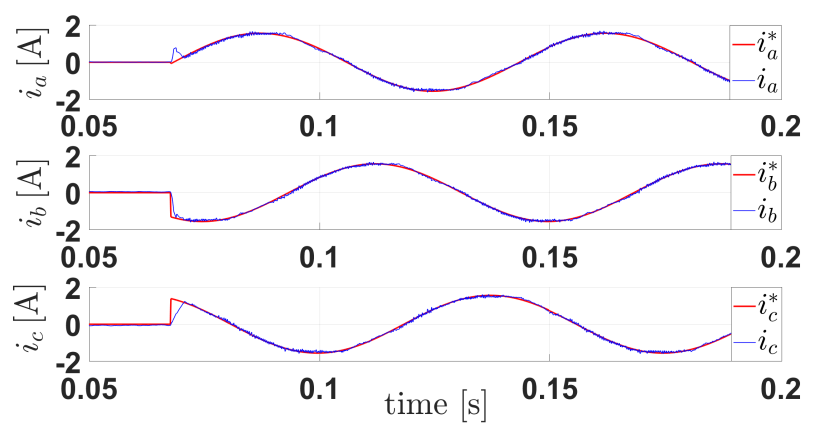

Fig. 11: $a b c$-current reference tracking $\left(f_{s}=24 \mathrm{kHz}, \bar{f}_{s w} \approx 2 \mathrm{kHz}\right.$, and $\lambda_{u}=0.0160, \operatorname{TDD}_{i} \approx 3.9 \%$ ).

load could not be tested, but the principle is easily extendable to the whole operating range.

A first series of current control tests at $400 \mathrm{rpm}$ constant speed, set by the PM are performed. During the tests the average dc-link was $300 \mathrm{~V}$ fixed by the dc-power supply, and the current references on the $d$ - and $q$-axis were set to $20 \%$ of the rated current. The single test duration is $3 \mathrm{~s}$ to acquire at least twenty electrical periods for an accurate computation of the TDD, whose trend is plotted over a wide range of switching frequencies, for three different values of sampling frequency: $f_{s}=16,24,32 \mathrm{kHz}$ (see Fig. 9). In the same figure, the current TDD produced by conventional FCS-MPC is also depicted. Similar to the simulation studies (see Fig. 5), the trend based on the experiments is the same, i.e., the proposed controller achieves better drive performance, as quantified by the current TDD. Note that the experimental current TDD is higher than the one acquired with the simulation tests due to the real-world non-idealities that exist in an experimental setup. In Figs. 10 and 11, the $d q$-current tracking and the phase-currents for the given operating point are shown.

Another series of tests for a different operating point were run. In this case the rotational speed was set to $100 \mathrm{rpm}$, the $d q$-axis current references were chosen to be equal to the rated current, while the dc-link voltage was set as was previously set to $300 \mathrm{~V}$. As for the previous set of tests, the trend illustrated in Fig. 12 proves that more favorable performance metrics can be demonstrated in the TDD for the given switching frequency.

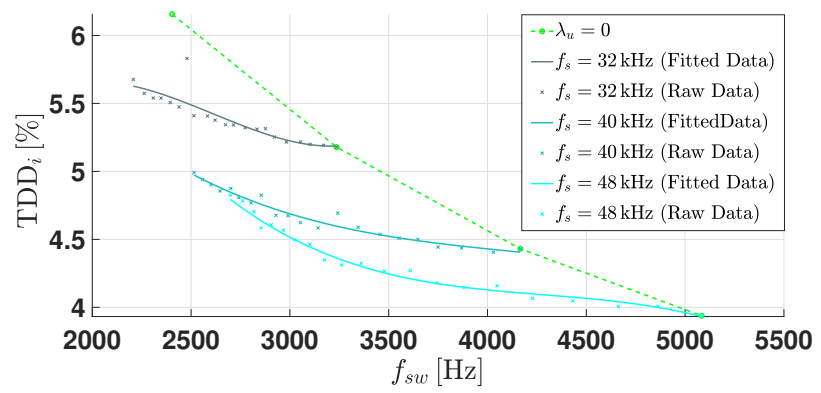

Fig. 12: Trade-off curve between current TDD and switching frequency $\left(i_{d}=\right.$ $\left.i_{q}=5.5 \mathrm{~A} ; \omega_{m}=100 \mathrm{rpm}, 0<\lambda_{u}<0.032\right)$.
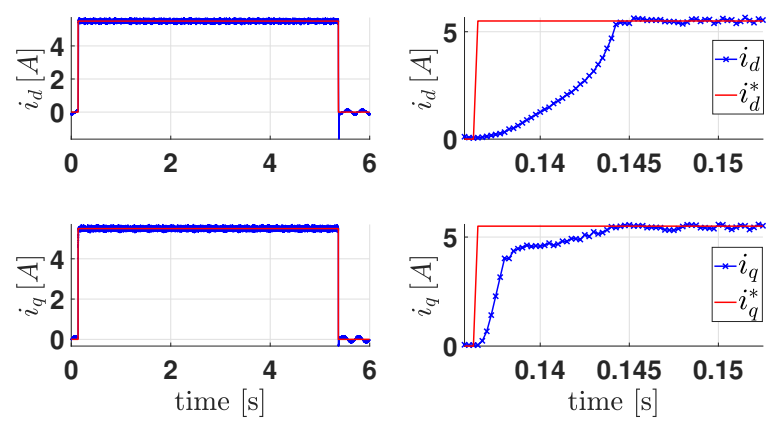

Fig. 13: $d q$-current reference tracking $\left(f_{s}=48 \mathrm{kHz}, \bar{f}_{s w} \approx 3.17 \mathrm{kHz}\right.$, and $\left.\lambda_{u}=0.0192\right)$.
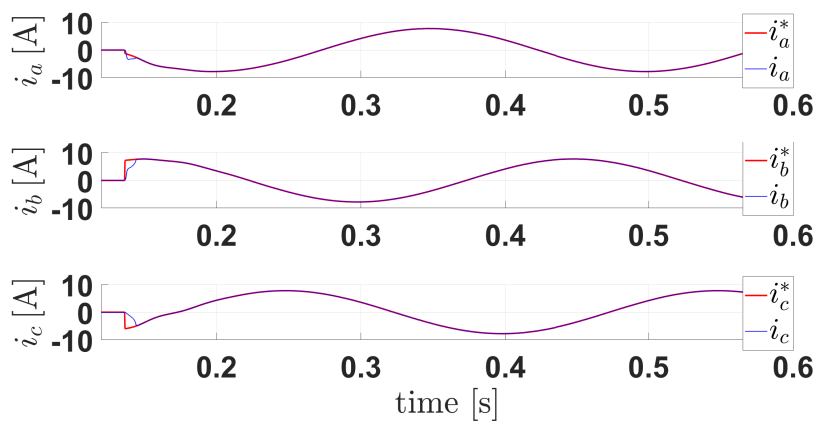

Fig. 14: $a b c$-current reference tracking $\left(f_{s}=48 \mathrm{kHz}, \bar{f}_{s w} \approx 3.17 \mathrm{kHz}\right.$, and $\lambda_{u}=0.0192, \mathrm{TDD}_{i} \approx 4.4 \%$ ).

Also in this case the $d q$-reference current tracking, and the phase currents are shown in Figs. 13 and 14, respectively. Notice that the control effort weighting factor selection does not have an analytical method to tune it, thus, the typical trialand-error procedure was considered to find suitable values to achieve the desired operating switching frequency.

\section{Conclusions And Future Work}

In this paper an FCS-MPC algorithm was introduced that achieves high granularity of switching. In doing so, the performance of a highly non-linear drive, such as the SyRel motor drive examined in this work, can be significantly improved, as quantified by the current TDD. Specifically, for a given switching frequency, lower TDD can be achieved when a 
relatively high granularity of switching is ensured. According to the presented results, this approach presents higher advantages at low-load operation. To further improve the tracking performance of the controller, an integrating element was added to the cost function. In doing so, system non-idealities and unmodeled dynamics are successfully addressed, and thus cannot affect the controller performance in an adverse manner, while the computational complexity of the FCS-MPC scheme remains the same. It is worth mentioning, however, that the tuning procedure for the control effort weighting factor still remains an open topic.

\section{REFERENCES}

[1] N. Bianchi, S. Bolognani, E. Carraro, M. Castiello, and E. Fornasiero, "Electric vehicle traction based on synchronous reluctance motors," IEEE Transactions on Industry Applications, vol. 52, no. 6, pp. 4762$4769,2016$.

[2] I. Nasui-Zah, A.-M. Nicorici, and C. Marțis, "Saturation and crosssaturation in synchronous reluctance machines," in 2018 International Conference and Exposition on Electrical And Power Engineering (EPE), 2018, pp. 0347-0351.

[3] S. Ferdous, P. Garcia, M. A. M. Oninda, and M. A. Hoque, "Mtpa and field weakening control of synchronous reluctance motor," in 2016 9th International Conference on Electrical and Computer Engineering (ICECE), 2016, pp. 598-601.

[4] G. H. B. Foo and X. Zhang, "Robust direct torque control of synchronous reluctance motor drives in the field-weakening region," IEEE Transactions on Power Electronics, vol. 32, no. 2, pp. 1289-1298, 2017.

[5] P. Karamanakos, E. Liegmann, T. Geyer, and R. Kennel, "Model predictive control of power electronic systems: Methods, results, and challenges," IEEE Open Journal of Industry Applications, vol. 1, pp. 95-114, 2020.

[6] P. Karamanakos and T. Geyer, "Guidelines for the design of finite control set model predictive controllers," IEEE Transactions on Power Electronics, vol. 35, no. 7, pp. 7434-7450, 2020.

[7] C.-K. Lin, J.-t. Yu, Y.-S. Lai, and H.-C. Yu, "Improved model-free predictive current control for synchronous reluctance motor drives," IEEE Transactions on Industrial Electronics, vol. 63, no. 6, pp. 39423953, 2016.

[8] R. Vargas, U. Ammann, and J. RodrÍguez, "Predictive approach to increase efficiency and reduce switching losses on matrix converters," IEEE Transactions on Power Electronics, vol. 24, no. 4, pp. 894-902, 2009.

[9] G. Cimini, D. Bernardini, A. Bemporad, and S. Levijoki, "Online model predictive torque control for permanent magnet synchronous motors," in 2015 IEEE International Conference on Industrial Technology (ICIT), 2015, pp. 2308-2313.

[10] L. Ortombina, E. Liegmann, P. Karamanakos, F. Tinazzi, M. Zigliotto, and R. Kennel, "Constrained long-horizon direct model predictive control for synchronous reluctance motor drives," in 2018 IEEE 19th Workshop on Control and Modeling for Power Electronics (COMPEL), 2018, pp. 1-8.

[11] J. Rodriguez, M. P. Kazmierkowski, J. R. Espinoza, P. Zanchetta, H. Abu-Rub, H. A. Young, and C. A. Rojas, "State of the art of finite control set model predictive control in power electronics," IEEE Transactions on Industrial Informatics, vol. 9, no. 2, pp. 1003-1016, 2013.

[12] S. Hanke, S. Peitz, O. Wallscheid, J. Böcker, and M. Dellnitz, "Finitecontrol-set model predictive control for a permanent magnet synchronous motor application with online least squares system identification," in 2019 IEEE International Symposium on Predictive Control of Electrical Drives and Power Electronics (PRECEDE), 2019, pp. 1-6.

[13] A. Favato, P. G. Carlet, F. Toso, and S. Bolognani, "A model predictive control for synchronous motor drive with integral action," in IECON 2018 - 44th Annual Conference of the IEEE Industrial Electronics Society, 2018, pp. 325-330.

[14] A. Borghese, A. P. Catalano, M. Riccio, L. Codecasa, A. Fayyaz, V. d'Alessandro, A. Castellazzi, L. Maresca, G. Breglio, and A. Irace, "An efficient simulation methodology to quantify the impact of parameter fluctuations on the electrothermal behavior of multichip SiC power modules," in Silicon Carbide and Related Materials 2018, ser. Materials Science Forum, vol. 963. Trans Tech Publications Ltd, 9 2019, pp. $855-858$.

[15] C. Scognamillo, A. P. Catalano, A. Borghese, M. Riccio, V. d'Alessandro, G. Breglio, A. Irace, R. N. Tripathi, A. Castellazzi, and L. Codecasa, "Electrothermal modeling, simulation, and electromagnetic characterization of a $3.3 \mathrm{kV} \mathrm{SiC}$ mosfet power module," in 2021 33rd International Symposium on Power Semiconductor Devices and ICs (ISPSD), 2021, pp. 123-126.

[16] E. Armando, R. I. Bojoi, P. Guglielmi, G. Pellegrino, and M. Pastorelli, "Experimental identification of the magnetic model of synchronous machines," IEEE Transactions on Industry Applications, vol. 49, no. 5, pp. 2116-2125, 2013.

[17] A. Galassini, G. Lo Calzo, A. Formentini, C. Gerada, P. Zanchetta, and A. Costabeber, "uCube: Control platform for power electronics," in 2017 IEEE Workshop on Electrical Machines Design, Control and Diagnosis (WEMDCD), 2017, pp. 216-221. 